\title{
Prehabilitation prior to lung cancer surgery: a small step forward
}

\author{
Andrew E. Giles, Sadeesh K. Srinathan \\ Section of Thoracic Surgery, Department of Surgery, Max Rady College of Medicine, University of Manitoba, Manitoba, Canada \\ Correspondence to: Sadeesh Srinathan, MD, MSc. GE-611, 820 Sherbrook St., Health Sciences Centre, Winnipeg, Manitoba R3A 1R9, Canada. \\ Email: ssrinathan@hsc.mb.ca. \\ Provenance: This is an invited article commissioned by the Section Editor Laura Chiara Guglielmetti (Cantonal Hospital Winterthur, Kantonsspital \\ Winterthur, Switzerland). \\ Comment on: Liu Z, Qiu T, Pei L, et al. Two-Week Multimodal Prehabilitation Program Improves Perioperative Functional Capability in Patients \\ Undergoing Thoracoscopic Lobectomy for Lung Cancer: A Randomized Controlled Trial. Anesth Analg 2019. [Epub ahead of print].
}

Submitted Nov 26, 2019. Accepted for publication Dec 11, 2019.

doi: $10.21037 /$ jtd.2019.12.54

View this article at: http://dx.doi.org/10.21037/jtd.2019.12.54

Ever since the National Emphysema Treatment Trial (1), it has been clear that preoperative pulmonary rehabilitation improves function in patients preparing to undergo lung resection. However, the ensuing supporting comparative literature has consisted of small randomized trials and moderately sized cohort studies with a variety of limitations (2). We are left with mixed impressions as to the role of prehabilitation in patients awaiting lung cancer surgery.

In response, Liu and colleagues (3) conducted a randomized controlled trial of unselected patients undergoing thoracoscopic lobectomy at a university hospital in Beijing for non-small cell lung cancer (NSCLC). Patients were randomized to usual medical care or to a homeadministered trimodality prehabilitation program (aerobic/ resistance/pulmonary exercises, nutrition, and psychological support) for two weeks prior to surgery. The primary endpoint was improvement in the six-minute walk distance (6MWD). Secondary outcomes were pulmonary function tests, quality of recovery, and physical and mental wellbeing. Concealment of allocation and blinding of outcome assessors both appeared to be satisfactory.

They show that after a median of 15 days of prehabilitation, patients walked 60 meters further on the $6 \mathrm{MWD}$ as compared to the control group at 30 days after surgery. Those in the experimental group had a 45-meter increase from baseline at the end of the program, just prior to surgery, compared to only 3.8 meters in the control arm. There were no differences in any of the secondary outcomes.

A previous systematic review (2) showed that improvement in recovery (reduced length of stay and overall complications) is possible after a prehabilitation program, albeit with high heterogeneity. While the present study achieved the minimum clinically important difference threshold of 30 meters [as determined among COPD patients undergoing rehab; (4)], it did not show a difference in clinically meaningful endpoints. Why this discrepancy? Certainly one answer is that it was not powered to do so, and with only 73 patients the likelihood of type II error is high.

It is also useful to note that the patient population differed substantially from that of other reports. Patients in this trial were young (mean 56 years old), female (70\%), with a normal-range BMI (mean BMI of 23). Baseline pulmonary function tests were near normal and $90 \%$ had never smoked. Only one patient had COPD, and few patient $(11 \%)$ were classified as ASA III or IV. Baseline 6MWD measured $560 \mathrm{~m}$, similar to healthy population averages (5).

So, what have they demonstrated? Primarily that a short but rigorous multimodal prehabilitation program can result in a measurable improvement in cardiopulmonary performance in generally young and fit patients. It is questionable whether this result will be replicated in a more heterogeneous population of patients with lung cancer, especially those seen in Europe and North America. It is possible that if a wider range of patients experience this degree of improvement, they may indeed have improved clinical outcomes. Alternatively, the typical patient may not be fit enough to begin with to benefit from this particular 
intervention.

The authors merit acknowledgement on several fronts. First, they addressed many of the methodologic issues of prior studies, such as concealment, adequate randomization and blinding of assessors. While not totally novel, the prehabilitation program was well considered, although quite demanding. The interventions were comprehensive, the course was abbreviated to 2 weeks to eliminate concerns associated with delay to oncologic resection (6), and home administration makes this widely applicable and potentially more affordable.

Thus, while their results do not provide further guidance regarding whether prehabilitation should be standard therapy for patients undergoing lobectomy for NSCLC, they do show us that methodologically sound studies on prehabilitation are possible. Second, that not all patients with lung cancer are comparable in terms of baseline function and comorbidity. A large-scale multinational RCT would be necessary to address the scope of this question and to ascertain which patients truly stand to benefit from prehabilitation. As to how this should be conducted, those designing such a trial would do well to consider the methods used here as a starting point.

\section{Acknowledgments}

None.

\section{Footnote}

Conflicts of Interest: The authors have no conflicts of interest to declare.

Cite this article as: Giles AE, Srinathan SK. Prehabilitation prior to lung cancer surgery: a small step forward. J Thorac Dis 2019;11(12):5664-5665. doi: 10.21037/jtd.2019.12.54
Ethical Statement: The authors are accountable for all aspects of the work in ensuring that questions related to the accuracy or integrity of any part of the work are appropriately investigated and resolved.

\section{References}

1. Ries AL, Make BJ, Lee SM, et al. The Effects of Pulmonary Rehabilitation in the National Emphysema Treatment Trial. Chest 2005;128:3799-809.

2. Sebio Garcia R, Yáñez Brage MI, Giménez Moolhuyzen $\mathrm{E}$, et al. Functional and postoperative outcomes after preoperative exercise training in patients with lung cancer: a systematic review and meta-analysis. Interact Cardiovasc Thorac Surg 2016;23:486-97.

3. Liu Z, Qiu T, Pei L, et al. Two-Week Multimodal Prehabilitation Program Improves Perioperative Functional Capability in Patients Undergoing Thoracoscopic Lobectomy for Lung Cancer: A Randomized Controlled Trial. Anesth Analg 2019. [Epub ahead of print].

4. Holland AE, Spruit MA, Troosters T, et al. An official European Respiratory Society/American Thoracic Society technical standard: field walking tests in chronic respiratory disease. Eur Respir J 2014;44:1428-46.

5. Zou H, Zhu X, Zhang J, et al. Reference equations for the six-minute walk distance in the healthy Chinese population aged 18-59 years. PLoS One 2017;12:e0184669.

6. Samson P, Patel A, Garrett T, et al. Effects of Delayed Surgical Resection on Short-Term and Long-Term Outcomes in Clinical Stage I Non-Small Cell Lung Cancer. Ann Thorac Surg 2015;99:1906-12; discussion 1913. 